\title{
THEORETICAL INVESTIGATION OF THE EFFECT OF CONJUATION OF THIOPHENE RINGS ON THE CHARGE TRANSPORT OF THIENOCORONENE DERIVATIVES
}

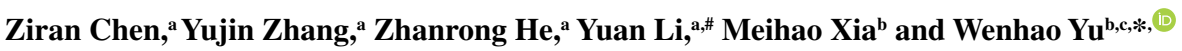 \\ aDepartment of Architecture and Environment Engineering, Sichuan Vocational and Technical College, Suining, Sichuan 629000, \\ P. R. China \\ ${ }^{b}$ College of Chemistry and Material Science, Sichuan Normal University, Chengdu, Sichuan 610068, P. R. China \\ ${ }^{\mathrm{c} C}$ College of Polymer Science \& Engineering, State Key Laboratory of Polymer Materials Engineering, Sichuan University, Chengdu, \\ 610065, P. R. China
}

Recebido em 04/06/2021; aceito em 24/08/2021; publicado na web em 15/09/2021

\begin{abstract}
Based on density functional theory, quantum chemical calculations of the charge-transport rates were performed for five disc-shaped coronene derivatives with varying numbers of fused thiophene rings, using different basis sets 6-31+G(d) and 6-311++G(d,p), hybrid functionals (B3LYP, M06-2X, CAM-B3LYP, WB97XD, M08-HX), and a dispersion-corrected hybrid functional (M06-2X+D3). Our results indicate that increasing the basis set and adding diffusion and polarisation functions had little effect on the molecular reorganisation energy, charge-transport matrix element $t$, and charge carrier mobility $\mu$. The charge carrier mobility calculated using B3LYP were relatively large, whereas the results calculated using CAM-B3LYP and WB97XD were similar. Among the five coronene derivatives, molecule $\mathbf{b}$ with one thiophene ring could be candidates for a $n$-type organic semiconductor, and molecule $\mathbf{c}$ with two thiophene rings can be designed as a $p$-type semiconductor.
\end{abstract}

Keywords: thiophene; coronene; charge carrier mobility; density functional theory; organic semiconductor.

\section{INTRODUCTION}

$\pi$-Conjugated polycyclic aromatic hydrocarbons (PAHs) are an important class of organic semiconductor materials with unique and tunable molecular structures, self-assembly behaviours, and photoelectric properties. ${ }^{1,2}$ They are widely used in fields such as organic field-effect transistors, organic light-emitting diodes, solar cells, chemical sensors, and solid electrolytes for lithium-ion batteries. Although numerous high-performance $p$-type organic semiconductor molecules have been synthesised, the synthesis of $n$-type organic semiconductor materials with high charge carrier mobility for application in electronic devices is still a challenge owing to the high-energy barrier of electron injection and the instability of organic anions in air. ${ }^{3}$ In the research and development of useful organic semiconductor materials, improving their electrical properties, especially their charge transfer rate, is critical. ${ }^{4}$ At the molecular level, a more compact arrangement among organic molecules and a higher order of molecular self-assembly generally correspond to higher charge carrier mobility. Therefore, the general molecular design is to expand the aromatic conjugate system, introduce heteroatoms into aromatic ring, replace the hydrogen atoms on the aromatic nucleus with fluorine atoms, and replace the benzene rings with thiophene rings to obtain organic semiconductor molecules with excellent performance. Thereby, the phase stability and stacking order of the semiconductor material are significantly improved, the charge transfer rate is increased, and the stability of these materials in the air and the service life of the related devices are improved.

The topological differences, hybridisation, and substituents on the molecular structure of PAHs can modulate their photoelectric properties. An important research direction for modifying the molecular structure of PAHs is the introduction of heteroatoms such as

*e-mail: yuwenhao@sicnu.edu.cn

\#alternative e-mail: 30278300@qq.com nitrogen, sulfur, selenium, and phosphorus atom into large conjugated $\pi$-electron systems, which can modify the polarity, electronic structure, and intermolecular interactions of the molecules, thereby modulating the physicochemical properties of these materials. Among PAHs, hexabenzocoronene and its derivatives exhibit rapid solid-state charge transfer owing to their high symmetry, high stability, and notable self-assembly ability. ${ }^{5-7}$ Thiophene and thiophene-fused PAHs have become important structural units for organic semiconductor materials owing to the extended orbitals of the sulfur atoms, which give rise to more efficient overlap of the intermolecular $\pi-\pi$ orbitals, enhanced intermolecular interactions, and higher charge carrier mobility. ${ }^{89}$ The synthesis of several thienocoronenes has been reported in the literature, including hexathienocoronenes, ${ }^{10,11}$ contorted dibenzotetrathienocoronenes, ${ }^{12}$ electron-poor dithienocoronene diimide, ${ }^{13}$ and thienoazacoronene, ${ }^{14,15}$ all of which have good self-assembly behaviour, good electrical properties, and potential applications in organic electronics. Unfortunately, the empirical charge transfer rates of large $\pi$-conjugated heteroatomic coronenes have only been reported in a few cases. For example, the transfer rate of dithienoazacoronene in thin-film field-effect transistors is nearly $0.028 \mathrm{~cm}^{2} \cdot \mathrm{v}^{-1} \cdot \mathrm{s}^{-1} \cdot{ }^{13}$

Several uncertainties are involved in determining the charge transfer properties of devices under experimental conditions. Furthermore, it is not possible to control and distinguish the various factors that affect the charge transfer rate. Theoretical computational simulations can be used to investigate the effects of certain factors on the charge transfer rate from a microscopic viewpoint and analyse the association between the geometric structure, crystal structure, and electronic structure of organic semiconductor materials and their electrical properties. ${ }^{16-21}$ Our group applied density functional theory to calculate the charge transfer rates of PAHs with different topological structures and different substituent groups or heteroatomisation to examine the relationship between the molecular structure and the charge-transport properties of different systems from the theoretical perspective. ${ }^{22-25}$ 
In this study, the 6-31+G(d) and 6-311++G(d,p) basis sets, B3LYP, M06-2X, CAM-B3LYP, WB97XD, and M08-HX hybrid functionals, and M06-2X+D3 dispersion-corrected hybrid functional are applied to the computational study of (1) the effects of increasing the basis set and the diffuse and polarisation functions on the charge-transport reorganisation energy, charge-transport matrix elements, and charge carrier mobility; (2) the merits of using different hybrid density functionals (B3LYP, M06-2X, CAM-B3LYP, WB97XD, M08-HX) and a dispersion-corrected hybrid functional (M06-2X+D3) to calculate the charge carrier mobility in large conjugated $\pi$-electron systems; (3) the effects of different thiophene ring orientations (molecules $\mathbf{c}$ and $\mathbf{d}$ are isomers) and quantity on the charge-transport reorganisation energy, charge-transport matrix elements, and charge carrier mobility. The structures of all the molecules are shown in Figure 1.

\section{THEORETICAL METHODOLOGY}

All calculations were performed using the Gaussian 16 Revision A.03 software package ${ }^{26}$ with the different basis set $6-31+G(d)$ and 6-311++G(d,p), hybrid functionals (B3LYP, M06-2X, CAMB3LYP,WB97XD, M08-HX), and dispersion-corrected hybrid functionals (M06-2X+D3). The different functionals and basis set has been used first to optimize the geometries of the ground state for neutral molecules and charged counterparts. The frequency calculations have been performed at the same level based on the optimized geometries. All the frequencies are real, showing that all the geometries obtained are energy minima on the potential energy surfaces. Subsequently, we had calculated the energy of highest occupied molecular orbital (HOMO) and of lowest unoccupied molecular orbital (LUMO), and the HOMO-LUMO energy gap of single-molecule optimized structure, the internal reorganization energies for hole and electron transfer $\left(\lambda_{+}\right.$, and $\lambda_{-}$, respectively), the charge transfer matrix element for hole and electron transfer $\left(t_{+}\right.$, and $t_{-}$, respectively) of the dimer. When a neutral molecule and a cation of coronene derivative molecule completely overlap, they form a dimer. Generally, the inter-disk spacing of PAH molecules is about $0.35 \mathrm{~nm},{ }^{10}$ but when the inter-disk spacing is set to be small, the two molecules stack up and down, often the surrounding substituents will overlap, which makes it impossible to form dimers effectively. Therefore, the distance of the centre of mass of the dimer molecule was set to $0.45 \mathrm{~nm}$. When the distance between the discs continues to increase, the intermolecular force gradually decreases, and the dimer binding energy tends to be stable. Small differences can be seen between the calculated intermolecular transfer matrix elements for the hole and electron. ${ }^{23}$

The mobility of charge carrier $\mu$ can be obtained using the Einstein equation, which is expressed as

$$
\mu=\mathrm{e} D /\left(\mathrm{k}_{\mathrm{B}} T\right)
$$

where e is the electron charge $\left(1.60 \times 10^{-19} \mathrm{C}\right), \mathrm{k}_{\mathrm{B}}$ is the Boltzmann constant $\left(1.38 \times 10^{-23} \mathrm{~J} / \mathrm{K}\right), T$ is the absolute temperature, and $D$ is the average diffusion coefficient of the charge starting from a molecule and towards all directions in three-dimensional space, as given by Formula (2). The diffusion coefficient is expressed as

$$
D=\frac{1}{6} \sum_{i} r_{i}^{2} k_{i} p_{i}
$$

where $r_{i}$ is the distance between adjacent molecules, $k_{i}$ is the charge transfer rate constant between adjacent molecules, and $p_{\mathrm{i}}$ is the probability

$$
p_{i}=k_{i} / \sum_{i} k_{i}
$$

of charge transfer to molecule $i$.

The $\pi$-conjugated organic semiconductor materials have the properties of one-dimensional charge carrier migration. The average diffusion coefficient can be simplified as

$$
D=\frac{1}{2} r^{2} k
$$

where $r$ is the disk spacing between adjacent discotic molecules, and $k$ is the charge transfer rate constant between adjacent molecules. The carrier mobility is obtained by substituting into formula (1):

$$
\mu=k \frac{r^{2}}{2 k_{B} T / e}
$$

According to the semi-classical model of Marcus charge transfer, ${ }^{27-31}$ the charge carrier mobility constant between adjacent molecules is expressed as the following formula:

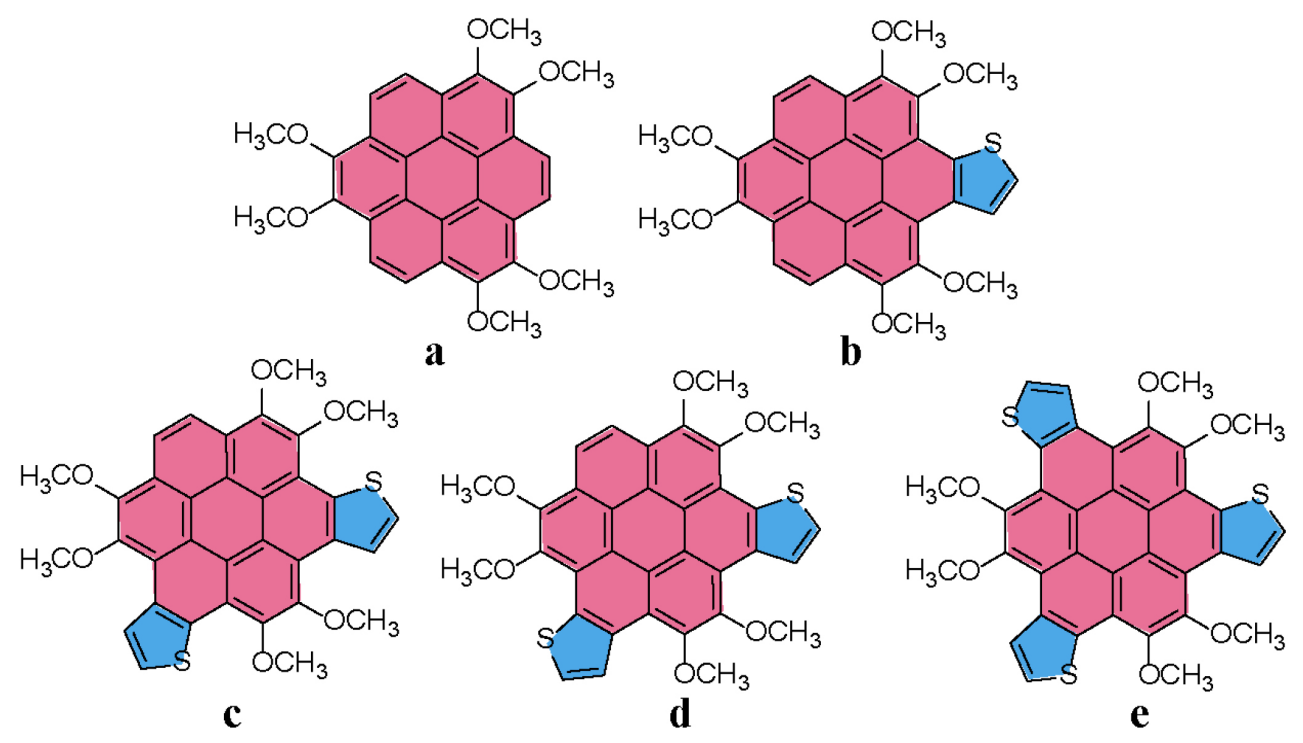

Figure 1. Molecular structures of coronene derivatives (a: coronene, $\boldsymbol{b}$ : monothienocoronene, $\boldsymbol{c}$ : dithienocoronene, $\boldsymbol{d}$ : isodithienocoronene, $\boldsymbol{e}:$ trithienocoronene) 


$$
k=\left(4 \pi^{2} / \mathrm{h}\right) t^{2}\left(4 \pi \lambda \mathrm{k}_{\mathrm{B}} T\right)^{-0.5} \exp \left[-\lambda /\left(4 \mathrm{k}_{\mathrm{B}} T\right)\right]
$$

where $h$ is the Planck constant $\left(6.626 \times 10^{-34} \mathrm{~J} \mathrm{~s}\right), t$ is the charge transfer matrix element, $\lambda$ is the charge reorganization energy, and $T$ is the absolute temperature. Under a certain temperature, $\lambda$ and $t$ are the main parameters affecting the charge carrier mobility constant. To achieve a larger charge transport rate constant, the molecule should have smaller reorganization energy $\lambda$ and larger intermolecular charge transport matrix element $t$.

The reorganization energy $\lambda$ mainly includes the internal reorganization energy $\left(\lambda_{\text {int }}\right)$ and the external reorganization energy $\left(\lambda_{\text {ext }}\right)$. The contribution of $\lambda_{\text {ext }}$ in crystals and amorphous films is very small, which usually is not considered, so we only calculated $\lambda_{\text {int }}$. The $\lambda_{\text {int }}$ is the energy change caused by the relaxation of the structure to a new equilibrium point after the ion becomes a neutral molecule and the neighboring neutral molecule becomes an ion during the charge transfer process. The hole reorganization energy $\left(\lambda_{+}\right)$and electron reorganization energy calculated by the adiabatic potential energy surface curve method $\left(\lambda_{-}\right)$are calculated as follows:

$$
\begin{aligned}
& \lambda_{+}=E\left(\mathrm{Ar}^{+} / \mathrm{Ar}\right)-E\left(\mathrm{Ar}^{+} / \mathrm{Ar}^{+}\right)+E\left(\mathrm{Ar} / \mathrm{Ar}^{+}\right)-E(\mathrm{Ar} / \mathrm{Ar}) \\
& \lambda_{-}=E(\mathrm{Ar} / \mathrm{Ar})-E\left(\mathrm{Ar}^{-} / \mathrm{Ar}^{-}\right)+E\left(\mathrm{Ar} / \mathrm{Ar}^{-}\right)-E(\mathrm{Ar} / \mathrm{Ar})
\end{aligned}
$$

where $E\left(\mathrm{Ar}^{-} / \mathrm{Ar}\right)$ and $E\left(\mathrm{Ar}^{+} / \mathrm{Ar}\right)$ are the energy of anions and cations in the neutral molecular geometry, respectively; $E\left(\mathrm{Ar}^{-} / \mathrm{Ar}^{-}\right)$and $E\left(\mathrm{Ar}^{+} / \mathrm{Ar}^{+}\right)$are the energy of anions and cations after optimized configuration; $E(\mathrm{Ar} / \mathrm{Ar})$ is the energy of the optimized neutral molecule; $E\left(\mathrm{Ar} / \mathrm{Ar}^{-}\right)$and $E\left(\mathrm{Ar} / \mathrm{Ar}^{+}\right)$are the energy of the neutral molecule in the anion and cation geometry, respectively.

In addition, the electron affinities, the ionization potential and the extraction potentials are related to the charge carrier transport properties. Thus, the vertical ionization potential $\left(\mathrm{IP}_{V}\right)$, the adiabatic ionization potential $\left(\mathrm{IP}_{a}\right)$, the vertical electron affinities $\left(\mathrm{EA}_{V}\right)$, the adiabatic electron affinities $\left(\mathrm{EA}_{a}\right)$, the hole extraction potentials (HEP), and the electron extraction potentials (EEP) were calculated by the following formulas: ${ }^{32,33}$

$$
\begin{aligned}
& \mathrm{IP}_{V} / \mathrm{IP}_{a}=E\left(\mathrm{Ar}^{+} / \mathrm{Ar}\right) / E\left(\mathrm{Ar}^{+} / \mathrm{Ar}^{+}\right)-E(\mathrm{Ar} / \mathrm{Ar}), \\
& \mathrm{EA}_{V} / \mathrm{EA}_{a}=E(\mathrm{Ar} / \mathrm{Ar}) / E\left(\mathrm{Ar}_{\mathrm{Ar}}\right)-E(\mathrm{Ar} / \mathrm{Ar}), \\
& \mathrm{HEP} / \mathrm{EEP}=E\left(\mathrm{Ar} / \mathrm{Ar}^{+}\right) / E(\mathrm{Ar} / \mathrm{Ar})-E\left(\mathrm{Ar}^{+} / \mathrm{Ar}^{+}\right) / E(\mathrm{Ar} / \mathrm{Ar}) .
\end{aligned}
$$

The charge transfer matrix element characterizes the coupling strength of electron-electron interaction, and several methods have been proposed to evaluate the transfer integral within a molecular dimer. The simplest way is the frontier orbit energy level splitting method. ${ }^{34,35}$ That is, the closed shell system was formed by adding one electron to the molecular/molecular cation system. The energy level splitting of HOMO and HOMO-1 at the transition state was calculated. One half is the hole transfer matrix element $t_{+}$. The closed shell system is formed by removing one electron from the molecular/ molecular anion system. The energy level splitting of LUMO and LUMO-1 at the transition state is calculated. One half is the negative charge transfer matrix element $t_{-}$. The transfer matrix elements for holes and electrons can be estimated by halving these values. The mean squares of the charge transfer matrix elements of the coronene derivatives were calculated using Equation (11) according to the Boltzmann distribution using the energies of the dimers at different dihedral angles $\left(E_{\mathrm{i}}\right)$ and the corresponding matrix elements $\left(t_{\mathrm{i}}\right)$ at a temperature of $298.15 \mathrm{~K}$. The root of $\left\langle t^{2}\right\rangle$ is the charge transfer matrix element, $t .{ }^{36}$ The carrier transport rates, $\mu$, were also calculated for the six molecules using Equation (5) and (6).

$$
n_{i=} \exp \left(-E_{i} / k \mathrm{~T}\right) / \Sigma_{j} \exp \left(-E_{j} / k T\right)
$$

$$
<t^{2}>=\Sigma_{i} n_{i} t_{i}^{2}=\Sigma_{i} t_{i}^{2} \exp \left(-E_{i} / k T\right) / \Sigma_{j} \exp \left(-E_{j} / k T\right)
$$

It is difficult to find the transition state of the charge transfer reaction, so almost all research work uses linear coordinates to describe the reaction process. The relationship between the $i$-th internal coordinate $\mathrm{Q}_{i}$ in the reaction and the reaction coordinate is: ${ }^{37,38}$

$$
Q_{i}=R Q_{i \mathrm{p}}+(1-R) Q_{i \mathrm{r}}
$$

$Q_{i \mathrm{p}}$ and $Q_{i \mathrm{r}}$ are the $i$-th internal coordinates of the product and reactant in equilibrium configuration, respectively. When $R=0$, the system is in the equilibrium configuration of reactants; when $R=1$, the system is in the equilibrium configuration of products; the reaction studied in this paper is a self-exchange reaction, and the transition state occurs at $R=0.5$.

\section{RESULTS AND DISCUSSION}

\section{Molecular design and structure optimisation}

The $6-31+\mathrm{G}(\mathrm{d})$ and $6-311++\mathrm{G}(\mathrm{d}, \mathrm{p})$ basis sets, the B3LYP, M06-2X, CAM-B3LYP, WB97XD, and M08-HX hybrid functionals, and the M06-2X+D3 dispersion-corrected hybrid functional were used for structure optimisation and frequency calculation for the five thiophene-containing coronene derivatives in Figure 1 to obtain stable structures. Molecules $\mathbf{c}$ and $\mathbf{d}$, containing two thiophene rings, are isomers and have the opposite orientation in the thiophene ring structure. The ball-and-stick models of the optimised molecules are shown in Figure 2. The stable structures obtained using the six methods of optimisation were relatively consistent. The five coronene derivatives adopted $C_{1}$-symmetric structures, and the dihedral angle between the thiophene and coronene rings was between $0.5^{\circ}$ and $2.6^{\circ}$; hence, the rigid nucleus formed by the thiophene and coronene rings was almost coplanar.

Based on the stable structures, the Natural Bond Orbital algorithm (NBO) method is used to calculate the five coronene derivatives $\mathbf{a} \sim \mathbf{e}$, and then the Natural Population Analysis (NPA) charge distribution of each molecule is analyzed. The results are shown in supplementary Table 1S. From the data in Table 1S, we can see that the introduction of different numbers of thiophene rings around the coronene ring has little effect on the total charge of the six oxygen atoms. However, the introduction of the thiophene ring increases the positive charge of the coronene ring, and with the increase of the number of thiophene rings, the positive charge of the coronene ring increases significantly. This indicates that the introduction of the thiophene ring can lead to an increase in the electron deficiency of the molecule, which is beneficial to the hole transport of the molecule.

\section{Frontier molecular orbitals}

The energy levels of the HOMO and LUMO directly affect the efficiency of hole or electron injection between the material and the electrode in device applications. The frontier orbital eigenenergy values of HOMO, HOMO-1, LUMO and LUMO+1 of the five thiophene-containing coronene derivatives and their intrinsic energies were calculated at the B3LYP/6-311++G(d,p) level of theory, as shown in Figure 3 and supplementary Figure 1S. The electron cloud distributions of HOMO and HOMO-1, LUMO and LUMO+1 of the five molecules are almost identical. They all belong to the bonding $\pi$ orbital and anti-bonding $\pi^{*}$ orbital, which will not change the nature of the electronic transition in the molecule. After introducing different numbers of thiophene rings into the molecules, the HOMO energy levels increased, with molecule $\mathbf{e}$ (containing three thiophene 


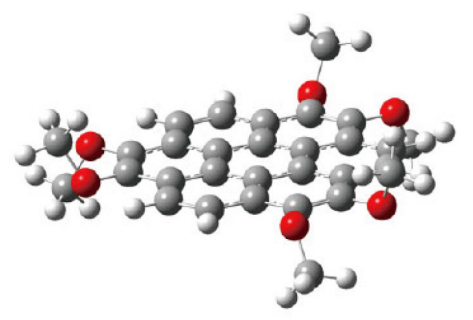

a

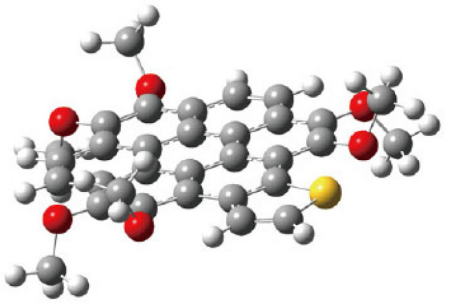

b

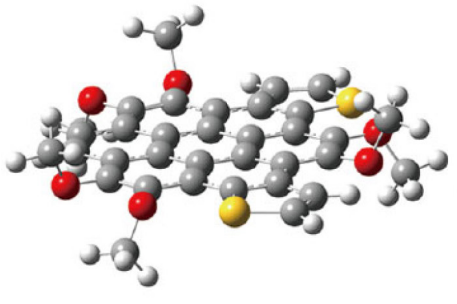

C

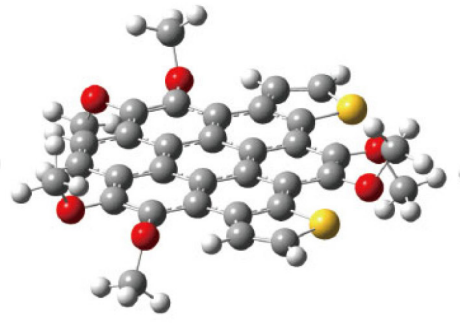

d

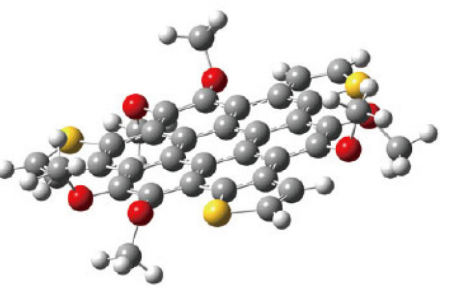

e

Figure 2. Optimized structures of coronene derivatives ( $\boldsymbol{a}$ : coronene, $\boldsymbol{b}$ : monothienocoronene, $\boldsymbol{c}$ : dithienocoronene, $\boldsymbol{d}$ : isodithienocoronene, $\boldsymbol{e}:$ trithienocoronene) using the B3LYP/6-31++G(d,p) method

rings) exhibiting the highest $\mathrm{HOMO}$ energy level at $-5.33 \mathrm{eV}$, which is close to the values obtained by electrochemical testing of similar structures ${ }^{39}$ whereas the LUMO energy levels decreased. Using the $6-311++\mathrm{G}(\mathrm{d}, \mathrm{p})$ basis set, all the results obtained by applying different calculation methods were highly similar (see Table $2 \mathrm{~S}$ and Figure $3 \mathrm{~S}$ ). For $n$-type organic semiconductors, reduction of the LUMO energy level is conducive to both efficient electron injection into the electrode and increased stability of the semiconductor in air. ${ }^{3,40}$ Molecules $\mathbf{b}$ and $\mathbf{c}$ had relatively low LUMO energy levels, indicating that they are conducive to electron injection. Comparison of molecules $\mathbf{c}$ and d, both of which contain two thiophene rings, indicated that the two thiophene rings in molecule $\mathbf{d}$ are arranged in the reverse order, and steric effects may have resulted in the slightly lower HOMO energy level, slightly higher LUMO energy level, and slightly larger energy gap of molecule d. Figure 3 demonstrates that the five coronene derivatives had similar HOMO and LUMO electron cloud density distributions on the coronene ring and the connected thiophene ring. The lone electron pairs on the oxygen atoms of the methoxy groups did not contribution to the HOMO and LUMO frontier molecular orbitals.

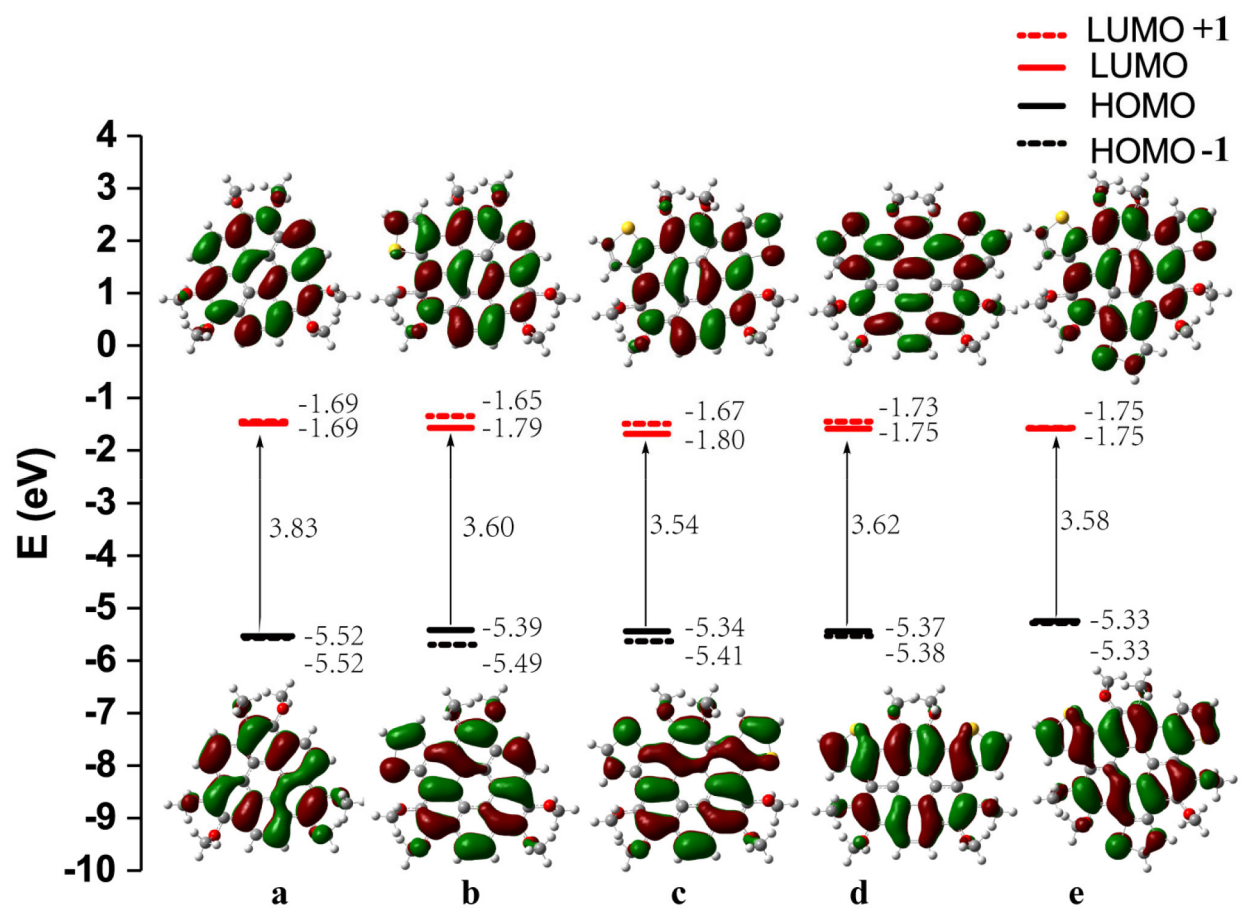

\section{Compounds}

Figure 3. LUMO +1 energies $\left(E_{L+1}\right)$, LUMO energies $\left(E_{L}\right)$, HOMO energies $\left(E_{H}\right)$, HOMO-1 energies $\left(E_{H-1}\right)$, and HOMO-LUMO gaps $($ Eg) of coronene derivatives $\boldsymbol{a} \sim \boldsymbol{e}$ at the B3LYP/6-311++G(d,p) level. The upper illustration is the ground state density plot of the LUMO, and the bottom illustration is the ground state density plot of the HOMO of the six molecules 
Table 1. The hole $\left(\lambda_{+}\right)$and electron $\left(\lambda_{-}\right)$reorganization energies $(\mathrm{kJ} / \mathrm{mol})$ of coronene derivatives $\mathbf{a} \sim \mathbf{e}$

\begin{tabular}{|c|c|c|c|c|c|c|c|}
\hline Comp. & $\lambda$ & $\begin{array}{c}\text { B3LYP/ } \\
6-31+G(d)\end{array}$ & $\begin{array}{c}\text { B3LYP/ } \\
6-311++G(d, p)\end{array}$ & $\begin{array}{l}\text { M06-2X/ } \\
6-31+G(d)\end{array}$ & $\begin{array}{c}\text { M06-2X/ } \\
6-311++G(d, p)\end{array}$ & $\begin{array}{l}\text { M08-HX/ } \\
6-31+\mathrm{G}(\mathrm{d})\end{array}$ & $\begin{array}{c}\text { M08-HX/ } \\
6-311++G(d, p)\end{array}$ \\
\hline \multirow{2}{*}{$\mathbf{a}$} & $\lambda_{+}$ & 52.09 & 53.22 & 62.51 & 65.22 & 66.93 & 68.82 \\
\hline & $\lambda_{-}$ & 24.75 & 24.88 & 30.18 & 30.11 & 28.59 & 27.77 \\
\hline \multirow{2}{*}{ b } & $\lambda_{+}$ & 36.24 & 36.66 & 56.18 & 57.21 & 57.94 & 57.94 \\
\hline & $\lambda_{-}$ & 22.51 & 22.87 & 27.82 & 27.75 & 26.38 & 26.09 \\
\hline \multirow{2}{*}{ c } & $\lambda_{+}$ & 33.6 & 34.09 & 45.98 & 47.02 & 46.99 & 48.86 \\
\hline & $\lambda_{-}$ & 21.44 & 21.77 & 26.49 & 26.91 & 25.44 & 25.49 \\
\hline \multirow{2}{*}{ d } & $\lambda_{+}$ & 39.56 & 40.18 & 49.24 & 50.54 & 50.77 & 49.99 \\
\hline & $\lambda_{-}$ & 21.64 & 21.86 & 26.26 & 26.62 & 25.10 & 24.91 \\
\hline \multirow{2}{*}{$\mathbf{e}$} & $\lambda_{+}$ & 28.59 & 29.21 & 36.39 & 37.02 & 29.5 & 29.39 \\
\hline & $\lambda_{-}$ & 19.66 & 20.09 & 24.47 & 24.87 & 23.26 & 23.01 \\
\hline
\end{tabular}

\section{Molecular reorganisation energy}

Using the $6-31+\mathrm{G}(\mathrm{d})$ and $6-311++\mathrm{G}(\mathrm{d}, \mathrm{p})$ basis sets, the B3LYP, M06-2X, and M08-HX density functionals were applied to optimise the calculated molecular and ionic energies, the ionic energies based on the molecular configuration, and the molecular energies based on the ionic configuration. The hole $\left(\lambda_{+}\right)$and electron $\left(\lambda_{-}\right)$transport reorganisation energies of the five thiophene-containing coronene derivatives were calculated using Equations (7) and (8), respectively, and are listed in Table 1, Figure 4S and Figure 5S (A and B). The difference between the hole and electron-transport reorganisation energies obtained by the same method for the different basis sets was small, thus indicating that increasing the diffuse and polarisation functions had little effect on the molecular reorganisation energies.

Figure 5S (C and D) shows the hole and electron-transport reorganisation energies obtained using the B3LYP, M06-2X, M06-2X+D3, CAM-B3LYP, WB97XD, and M08-HX functionals using the $6-311++\mathrm{G}(\mathrm{d}, \mathrm{p})$ basis set. It can be seen from Figure $5 \mathrm{~S}$ that for the same molecule with the basis set unchanged, the results calculated using the B3LYP and M08-HX functionals were relatively small, whereas the hole and electron-transport reorganisation energies obtained with the M06-2X, M06-2X+D3, CAM-B3LYP, and WB97XD functionals were close to each other. This shows that the introduction of DFT-D3 dispersion correction had little effect on the reorganisation energy. The results for molecules $\mathbf{c}$ and $\mathbf{d}$ were similar, which also indicates that the orientation of the thiophene rings in the molecule had little effect on the reorganisation energy of the molecule. The hole-transport reorganisation energies $\left(\lambda_{+}\right)$of the five molecules were significantly larger than their electron-transport reorganisation energies $\left(\lambda_{-}\right)$, which suggests that their electron-transport performance is better than their hole-transport performance.

The ionisation potential (IP) and electron affinity (EA) of a molecule are important parameters for characterising its hole and electron injection ability. Whether electrons can be efficiently injected into the LUMO energy level depends on the level of the EA; to achieve an excellent $n$-type organic semiconductor, the molecule must have a large EA. Conversely, for holes to be efficiently injected into the HOMO energy level, the IP must be sufficiently small. ${ }^{41}$ Table 2 shows the IPs, EAs, and extraction potentials calculated at the CAM-B3LYP/6-311++G(d,p) level of theory, while Table 3S and Table $4 \mathrm{~S}$ shows the IPs, EAs, and extraction potentials calculated at the B3LYP/6-311++G(d,p) and WB97XD/6-311++G(d,p) level of theory, respectively. After introducing different numbers of thiophene rings into the parent 1,2,5,6,9,10-hexa(methoxy)coronene (molecule a), the IPs of the molecules decreased, whereas the EAs increased, except for molecule $\mathbf{d}$.
Table 2. Ionization potentials (IP/ev), electron affinities (EA/ev) and extraction potentials of the studied molecules (all in electronvolts) at the basis set CAM-B3LYP/6-311++G(d,P)level.

\begin{tabular}{ccccccc}
\hline Comp. & $\mathrm{IP}_{V}$ & $\mathrm{IP}_{a}$ & $\mathrm{HEP}$ & $\mathrm{EA}_{V}$ & $\mathrm{EA}_{a}$ & $\mathrm{EEP}$ \\
\hline $\mathbf{a}$ & 6.8322 & 6.5874 & -6.2483 & -0.4805 & -0.6394 & 0.7957 \\
$\mathbf{b}$ & 6.6260 & 6.5045 & -6.4330 & -0.5983 & -0.7425 & 0.8859 \\
$\mathbf{c}$ & 6.7634 & 6.5546 & -6.3148 & -0.5034 & -0.6839 & 0.8357 \\
$\mathbf{d}$ & 6.7980 & 6.5801 & -6.2920 & -0.4665 & -0.6196 & 0.7707 \\
e & 6.7385 & 6.5557 & -6.3441 & -0.5049 & -0.6473 & 0.7886 \\
\hline
\end{tabular}

$\mathrm{IP}_{V}$ : vertical ionization potentials, $\mathrm{IP}_{a}$ : adiabatic ionization potentials, HEP: hole extraction potentials, $\mathrm{EA}_{V}$ : vertical electron affinities, EA $\mathrm{A}_{a}$ adiabatic electron affinities, EEP: electron extraction potentials.

\section{Charge-transport matrix elements and charge-transport rate constants}

Figure 4A an 4B shows the charge-transport matrix element $t$ calculated by applying the B3LYP, M06-2X, and M08-HX density functionals using the $6-31+\mathrm{G}(\mathrm{d})$ and $6-311++\mathrm{G}(\mathrm{d}, \mathrm{p})$ basis sets, respectively. The figure shows that increasing both the basis set and the diffuse and polarisation functions did not have a significant impact on the charge-transport matrix element $t$. Figure 4 (C, D) shows the charge-transport matrix elements calculated using the B3LYP, M06-2X, M06-2X+D3, CAM-B3LYP, WB97XD, and M08-HX functionals with the same basis set $6-311++G(d, p)$. It can be seen from the figure that for each molecule, the results calculated using the B3LYP and M08-HX functionals deviated significantly from each other, whereas the results obtained using the other four functionals were similar. In particular, the results obtained with M06-2X+D3, CAM-B3LYP, and WB97XD were similar, indicating that increasing the dispersion correction was more favourable for calculation of the charge-transport matrix elements.

The charge-transport rate constant $k$ and carrier mobility $\mu$ of the five molecules were calculated from Equation (6) and Equation 1, respectively. Table $5 \mathrm{~S}$ shows the charge-transport rate constant $k$ of the five molecules. The data indicate that introducing thiophene rings into the coronene with molecule $\mathbf{a}$ as the parent facilitated an increase in the charge-transport performance in all the cases. However, the charge-transport performance did not increase with the number of thiophene rings added, as the charge-transport performance did not improve significantly after adding two and three thiophene rings compared to the parent (molecule a); the orientation of the thiophene rings also had little effect on the charge-transport performance. 

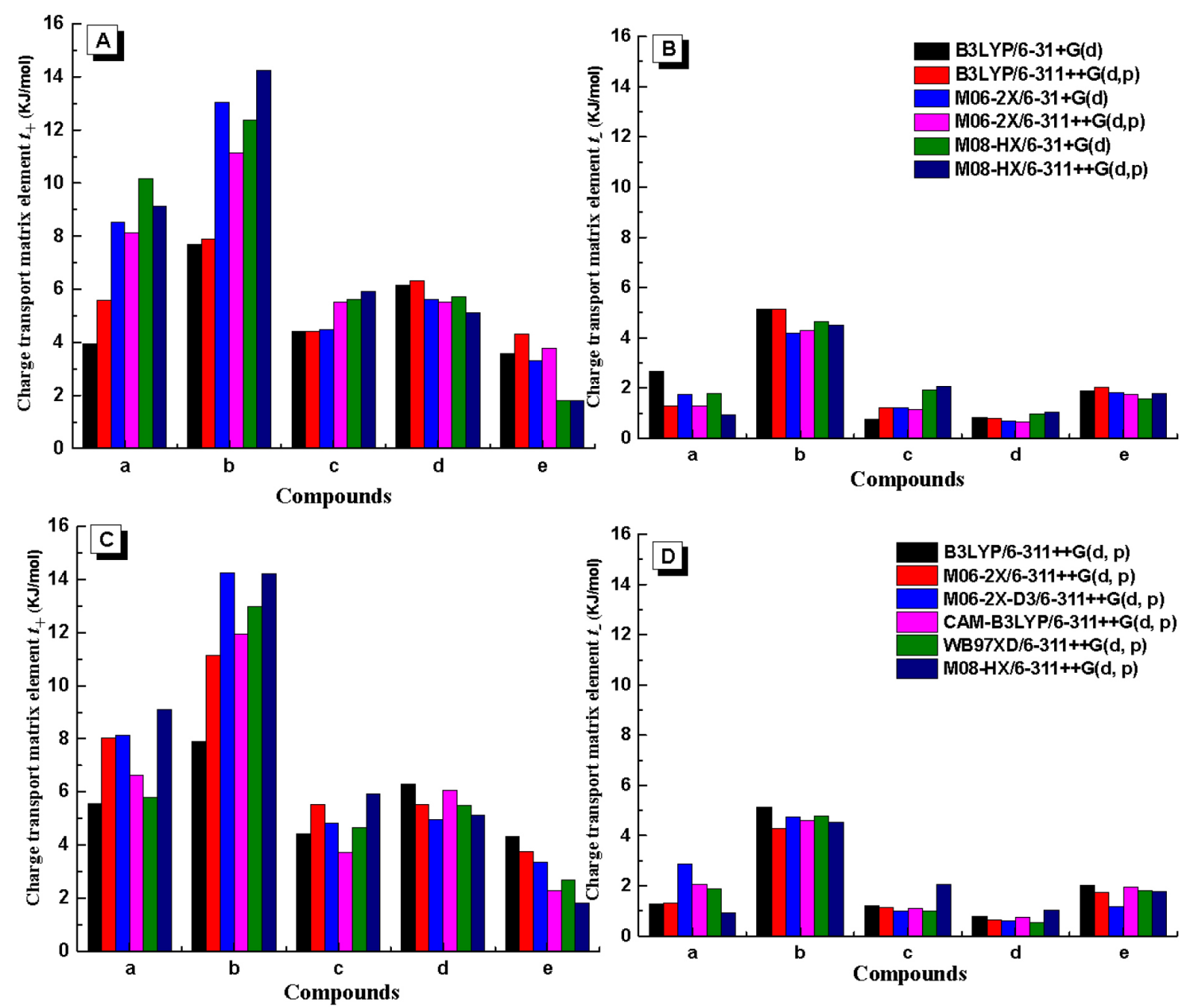

Figure 4. Charge transport matrix element $t_{+}(\mathrm{mev} / \mathrm{mol})(A)(B)$ at the basis set $6-31+G(d)$ or $6-311++G(d$, $p)$ level, Charge transport matrix element $t_{-}($mev/ $m o l)(C)(D)$ using six different hybrid functionalsat the same basis set $6-311++G(d, p)$ level

\section{Charge carrier mobility}

Table 3 and Figure 5 show the charge carrier mobility values obtained using different basis sets and different density functionals. The results show that changing the basis set did not have a significant effect on the charge carrier mobility. In addition, calculated using B3LYP were significantly larger, whereas the results of the other methods were more similar, especially those obtained using the
M06-2X, CAM-B3LYP, and WB97XD functionals. This is consistent with our previous research findings.

Table 4 show the charge-transport reorganisation energy, chargetransport matrix element, and charge carrier mobility of the five coronene derivatives calculated using the $6-311++\mathrm{G}(\mathrm{d}, \mathrm{p})$ basis set and the M06-2X, CAM-B3LYP, and WB97XD functionals. The hole and electron-transport reorganisation energies $\left(\lambda_{+}\right.$and $\left.\lambda_{-}\right)$were reduced by introducing different numbers of thiophene rings onto the coronene

Table 3. Charge carrier mobility $\mu\left(\mathrm{cm}^{2} \mathrm{v}^{-1} \mathrm{~s}^{1}\right)$ of a-e the B3LYP/6-31+G(d), B3LYP/6-311++G(d, p), M06-2X/6-31+G(d), M06-2X/6-311++G(d,p), M08-HX/6-31+G(d) and M08-HX/6-311++G(d,p) level. Dimer distance: $0.45 \mathrm{~nm}$

\begin{tabular}{|c|c|c|c|c|c|c|c|}
\hline Comp. & $\mu$ & $\begin{array}{c}\text { B3LYP/ } \\
6-31+\mathrm{G}(\mathrm{d})\end{array}$ & $\begin{array}{c}\text { B3LYP/ } \\
\text { 6-311++G(d,p) }\end{array}$ & $\begin{array}{c}\text { M06-2X/ } \\
6-31+G(d)\end{array}$ & $\begin{array}{c}\mathrm{M} 06-2 \mathrm{X} / \\
6-311++\mathrm{G}(\mathrm{d}, \mathrm{p})\end{array}$ & $\begin{array}{l}\text { M08-HX/ } \\
6-31+\mathrm{G}(\mathrm{d})\end{array}$ & $\begin{array}{c}\mathrm{M} 08-\mathrm{HX} / \\
6-311++\mathrm{G}(\mathrm{d}, \mathrm{p})\end{array}$ \\
\hline \multirow{2}{*}{$\mathbf{a}$} & $\mu_{+}$ & 0.0234 & 0.0142 & 0.0121 & 0.0079 & 0.0107 & 0.007 \\
\hline & $\mu_{-}$ & 0.0888 & 0.0183 & 0.0189 & 0.0103 & 0.0231 & 0.007 \\
\hline \multirow{2}{*}{ b } & $\mu_{+}$ & 0.1799 & 0.181 & 0.0561 & 0.0366 & 0.0458 & 0.047 \\
\hline & $\mu_{-}$ & 0.4014 & 0.385 & 0.1405 & 0.1495 & 0.2048 & 0.2009 \\
\hline \multirow{2}{*}{ c } & $\mu_{+}$ & 0.0797 & 0.076 & 0.0203 & 0.0276 & 0.0287 & 0.0259 \\
\hline & $\mu_{-}$ & 0.0098 & 0.024 & 0.0142 & 0.0118 & 0.0395 & 0.0456 \\
\hline \multirow{2}{*}{ d } & $\mu_{+}$ & 0.0788 & 0.0773 & 0.0223 & 0.0276 & 0.0196 & 0.0172 \\
\hline & $\mu_{-}$ & 0.0111 & 0.0105 & 0.0044 & 0.0039 & 0.0105 & 0.0125 \\
\hline \multirow{2}{*}{ e } & $\mu_{+}$ & 0.0951 & 0.127 & 0.0324 & 0.0394 & 0.0214 & 0.0221 \\
\hline & $\mu_{-}$ & 0.0786 & 0.085 & 0.0399 & 0.0343 & 0.0335 & 0.0457 \\
\hline
\end{tabular}



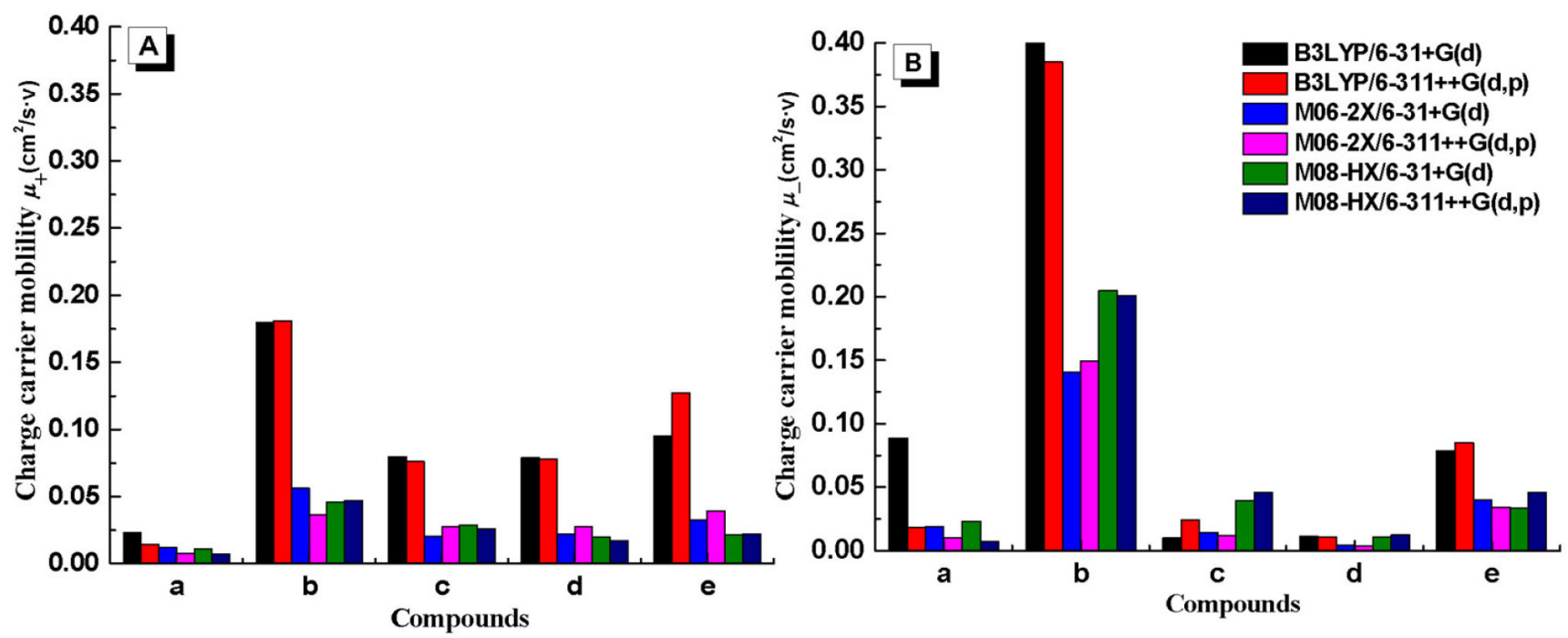

Figure 5. Charge carrier mobility (A) hole $\mu_{+}$and $(B)$ electron $\mu_{-}\left(\mathrm{cm}^{2} \cdot v^{-1} \cdot \mathrm{s}^{I}\right)$ of five coronene derivatives using three different functionals $(B 3 L Y P-M 06-2 X$ and M08-HX) at the different basis set level

ring of the parent (molecule a). Molecule b (containing one thiophene ring) showed increases in both the hole and electron-transport matrix elements, and the joint action of the two species led to the hole mobility $\mu_{+}$being 9.8 times that of molecule a, an electron mobility $\mu_{-}$that is 6.7 times that of molecule a. Moreover, electron mobility $\mu_{-}$that is 2.4 times the hole mobility $\mu_{+}$. Hence, molecule $\mathbf{b}$ is more favourable for electron-transport. Compared to the parent (molecule a), molecules $\mathbf{c}$ and $\mathbf{d}$ (containing two thiophene rings) showed a larger margin of decrease in the hole-transport reorganisation energy $\lambda_{+}$ compared to the electron-transport reorganisation energy $\lambda_{-}$. Whereas the hole-transport matrix element $t_{+}$decreased by a smaller margin than the electron-transport matrix element $t_{-}$, and the hole mobility $\mu_{+}$ was 2.4 times the electron mobility $\mu_{-}$, thus favouring hole-transport. Molecules $\mathbf{c}$ and $\mathbf{d}$ showed similar hole mobility $\mu_{+}$and electron mobility $\mu_{-}$, indicating that the orientation of the thiophene rings had little effect on the charge carrier mobility. Compared with the parent (molecule a), the hole-transport reorganisation energy $\lambda_{+}$and electron-transport reorganisation energy $\lambda$ _of molecule $\mathbf{e}$ (containing three thiophene rings) were reduced by 40 and $17 \%$, respectively, thus indicating that the hole-transport reorganisation energy $\lambda_{+}$decreased by a larger margin than the electron-transport reorganisation energy $\lambda$. While the hole-transport matrix element $t_{+}$was reduced by $60 \%$, whereas the electron-transport matrix element $t_{-}$was reduced by only $5 \%$, causing the electron mobility $\mu_{-}$to be 2.5 times higher than the hole mobility $\mu_{+}$, which is more favourable for electron transport.

To further confirm the patterns of variation in the electrontransport properties of the five molecules studied, the centroid distance of the molecular and anionic dimer was adjusted to $0.42 \mathrm{~nm}$ for molecule a and $0.40 \mathrm{~nm}$ for molecules $\mathbf{b}, \mathbf{c}, \mathbf{d}$, and e. Thereafter, the M06-2X/6-311++G(d,p), CAM-B3LYP/6-311++G(d,p), and WB97XD/6-311++G(d,p) functionals were used to calculate the electron-transport carrier mobility values of the five coronene derivatives. The three curves obtained using the three methods had similar shapes and exhibited the same trends. The molecule with the best electron-transport property was molecule $\mathbf{b}$, followed by molecule $\mathbf{e}$, and the worst electron transport was observed for molecule d.

In summary, the introduction of one and three thiophene rings on the coronene ring of the parent (molecule a) led to a greater electron mobility $\mu_{-}$than hole mobility $\mu_{+}$. This was especially the case for molecule $\mathbf{b}$, for which the electron mobility $\mu_{-}$was significantly larger than the hole mobility $\mu_{+}$, and hence, molecule $\mathbf{b}$ can be designed as an $n$-type organic semiconductor material that is favourable to electron transport. Molecules $\mathbf{c}$ and $\mathbf{d}$ (containing two thiophene rings) can be designed as $p$-type organic semiconductor materials that are conducive to hole transport as their hole mobility $\mu_{+}$is greater than their electron mobility $\mu_{-}$.

\section{CONCLUSIONS}

Using different basis sets 6-31+G(d) and 6-311++G(d,p), hybrid functionals (B3LYP, M06-2X, CAM-B3LYP, WB97XD, M08-HX), and a dispersion-corrected hybrid functional (M06-2X+D3), quantum chemical calculations of the charge-transport rates

Table 4. Reorganization energies $\lambda$, Charge transport matrix element $t\left(\mathrm{~kJ} \mathrm{~mol}^{-1}\right)$, charge carrier mobility $\mu\left(\mathrm{cm}^{2} \mathrm{v}^{-1} \mathrm{~s}^{1}\right)$ of a-e the CAM-B3LYP/6-311++G(d, p) and WB97XD/6-311++G(d, p) level

\begin{tabular}{|c|c|c|c|c|c|c|c|c|c|c|c|c|}
\hline Comp. & $\lambda$ & M06-2X & CAM-B3LYP & WB97XD & $t$ & M06-2X & CAM-B3LYP & WB97XD & $\mu$ & M06-2X & CAM-B3LYP & WB97XD \\
\hline \multirow[b]{2}{*}{$\mathbf{a}$} & $\lambda_{+}$ & 65.22 & 63.01 & 59.33 & $t_{+}$ & 8.02 & 6.64 & 5.81 & $\mu_{+}$ & 0.0079 & 0.007 & 0.0079 \\
\hline & $\lambda_{-}$ & 30.11 & 32.88 & 32.95 & $t_{-}$ & 1.29 & 2.06 & 1.88 & $\mu_{-}$ & 0.0103 & 0.019 & 0.0156 \\
\hline \multirow{2}{*}{ b } & $\lambda_{+}$ & 57.21 & 52.86 & 56.76 & $t_{+}$ & 11.13 & 11.95 & 12.97 & $\mu_{+}$ & 0.0366 & 0.068 & 0.0534 \\
\hline & $\lambda$ & 27.75 & 30.41 & 30.68 & $t_{-}$ & 4.28 & 4.61 & 4.79 & $\mu_{-}$ & 0.1495 & 0.127 & 0.1323 \\
\hline \multirow[b]{2}{*}{ c } & $\lambda_{+}$ & 47.02 & 43.28 & 48.82 & $t_{+}$ & 5.52 & 3.72 & 4.66 & $\mu_{+}$ & 0.0276 & 0.019 & 0.0161 \\
\hline & $\lambda$ & 26.91 & 29.45 & 29.94 & $t_{-}$ & 1.14 & 1.09 & 0.99 & $\mu_{-}$ & 0.0118 & 0.008 & 0.0063 \\
\hline \multirow{2}{*}{ d } & $\lambda_{+}$ & 50.54 & 48.83 & 52.01 & $t_{+}$ & 5.52 & 6.05 & 5.48 & $\mu_{+}$ & 0.0276 & 0.027 & 0.0158 \\
\hline & $\lambda_{-}$ & 26.62 & 29.35 & 29.67 & $t_{-}$ & 0.64 & 0.75 & 0.55 & $\mu_{-}$ & 0.0039 & 0.004 & 0.0019 \\
\hline \multirow[b]{2}{*}{$\mathbf{e}$} & $\lambda_{+}$ & 37.02 & 38.06 & 39.08 & $t_{+}$ & 3.76 & 2.29 & 2.68 & $\mu_{+}$ & 0.0394 & 0.013 & 0.0105 \\
\hline & $\lambda_{-}$ & 24.87 & 27.38 & 28.19 & $t_{-}$ & 1.73 & 1.96 & 1.81 & $\mu_{-}$ & 0.0343 & 0.033 & 0.0252 \\
\hline
\end{tabular}


were performed for five derivatives of the disc-shaped molecule 1,2,5,6,9,10-hexa(methoxy)coronene with varying numbers of fused thiophene rings. Molecular structure optimisation shows that the rigid nuclei of the five molecules are quasi-planar, large, $\pi$-conjugated aromatic ring systems. Increasing the basis set and adding diffusion and polarisation functions had little effect on the molecular reorganisation energy, charge-transport matrix element $t$, and charge carrier mobility $\mu$. The charge carrier mobility $\mu$ values calculated using B3LYP were relatively large, whereas the results calculated using CAM-B3LYP and WB97XD were similar. Among the five coronene derivatives, molecule $\mathbf{b}$ (containing one thiophene ring) showed the largest hole mobility $\mu_{+}$and electron mobility $\mu_{-}$, where the electron mobility $\mu_{-}$was 2.4 times its hole mobility $\mu_{+}$; this molecule is more favourable for electron transport, and hence, it can be used as an $n$-type organic semiconductor material. Molecule c (containing two thiophene rings) can be designed as a $p$-type semiconductor material as its hole mobility $\mu_{+}$is greater than its electron mobility $\mu_{-}$. It is anticipated that the results of this study will facilitate the experimental synthesis of sulfur heteroatom-hybridised organic semiconductor compounds with excellent electrical properties.

\section{SUPPLEMENTARY MATERIAL}

Figures 1S-5S and Tables 1S-5S are freely available at http:// quimicanova.sbq.org.br, in pdf format.

\section{ACKNOWLEDGEMENTS}

This work was supported by the Project of Science and Technology Department of Sichuan Province (Grant Number 2015GZ0343), the Key Project of Sichuan Provincial Department of Education (grant number 16ZB0059, 17ZA0346).

\section{REFERENCES}

1. Kumar, S.; Chem. Soc. Rev. 2006, 35, 83.

2. Wohrle, T.; Wurzbach, I.; Kirres, J.; Kostidou, A.; Kapernaum, N.; Litterscheidt, J.; Haenle, J. C.; Staffeld, P.; Baro, A.; Giesselmann, F.; Laschat, S.; Chem. Rev. 2016, 116, 1139.

3. Zhou, K.; Dong, H.-L.; Zhang, H.-L.; Hu, W.-P.; Phys. Chem. Chem. Phys. 2014, 16, 22448.

4. Bushby, R.-J.; Kelly, S.-M.; O’Neill, M.; Liquid Crystalline Semiconductors: Materials, Properties and Applications, Springer Science \& Business Media. 2012.

5. Ito, S.; Wehmeier, M.; Brand, J.-D.; Kübel, C.; Epsch, R.; Rabe, J.-P.; Müllen, K.; Chem. Eur. J. 2000, 6, 4327.

6. Simpson, C.-D.; Wu, J.-S.; Watson, M.-D.; Müllen, K.; J. Mater. Chem. 2004, 14, 494.

7. Kumar, S.; Tao, Y.-T.; Chem. - Asian J. 2021, 16, 621.

8. Mishra, A.; Ma, C.-Q.; Bäuerle, P.; Chem. Rev. 2009, 109, 1141.

9. Cinar, M.-E.; Ozturk, T.; Chem. Rev. 2015, 115, 3036.

10. Chen, L.; Puniredd, S.-R.; Tan, Y.-Z.; Baumgarten, M.; Zschieschang, U.; Enkelmann, V.; Pisula, W.; Feng, X.; Klauk, H.; Müllen, K.; J. Am. Chem. Soc. 2012, 134, 17869.

11. Chen, L.; Mali, K.-S.; Puniredd, S.-R.; Baumgarten, M.; Parvez, K.; Pisula, W.; De Feyter, S.; Mullen, K;. J. Am. Chem. Soc. 2013, 135, 13531.

12. Chiu, C.-Y.; Kim, B.; Gorodetsky, A.-A.; Sattler, W.; Wei, S.-J.; Sattler, A.; Steigerwald, M.; Nuckolls, C.; Chem. Sci. 2011, 2, 1480.

13. Li, Y.; Xu, W.; Motta, S.-D.; Negri, F.; Zhu, D.-B.; Wang, Z. -H.; Chem. Comm. 2012, 48, 8204.

14. He, B.; Pun, A.-B.; Klivansky, L.-M.; McGough, A.-M.; Ye, Y.-F.; Zhu, J.-F.; Guo, J.-H.; Teat, S. J.; Liu, Y.; Chem. Mater. 2014, 26, 3920.
15. He, B.; Dai, J.; Zherebetskyy, D.; Chen, T.-L.; Zhang, B.-A.; Teat, S.-J.; Zhang, Q.-C.; Wang, L.-W.; Liu, Y.; Chem. Sci. 2015, 6, 3180.

16. Ma, Y.; Zhao, C.-B.; Wang, W.-L.; Yin, S.-W.; Wei, J.-F.; Sci. Chin. Chem. 2013, 43, 1172.

17. Yin, J.; Chaitanya, K.; Ju, X.-H.; Comput. Theor. Chem. 2015, 1062, 56.

18. Chai, S.; Huang, J.-D.; Comput. Theor. Chem. 2015, 1069, 48.

19. Sahoo, S.-R.; Sahu, S.; Sharma, S.; J. Phys. Org. Chem. 2017, e3785.

20. Volpi, R.; Camilo, A.-C.-S.; Filho, D.; Navarrete, J.-T.-L.; Gomez-Lor, B.; Delgado, M.-C.-R.; Linares, M.; Phys. Chem. Chem. Phys. 2017, 19, 24202.

21. Oshi, R.; Abdalla, S.; Springborg, M.; Comput. Theor. Chem. 2018, 1128,60 .

22. Chen, Z.-R.; Yu, W,-H.; Li, Q.; Chin. J. Struct. Chem. 2012, 31, 851.

23. Chen, Z.-R.; He, Z.-R.; Xu, Y.-H.; Yu, W.-H.; J. Chem. Sci. 2017, 129, 1341.

24. Chen, Z.-R.; Li, Y.; He, Z.-R.; Xu, Y.-H.; Yu, W.-H.; J. Chem. Res. 2019 , $48,293$.

25. Chen, Z.-R.; Yu, W,-H.; Mol. Phys. 2017, 115, 424.

26. Frisch, M. J.; Trucks, G. W.; Schlegel, H. B.; Scuseria, G. E.; Robb, M. A.; Cheeseman, J. R.; Scalmani, G.; Barone, V.; Petersson, G. A.; Nakatsuji, H.; Li, X.; Caricato, M.; Marenich, A. V.; Bloino, J.; Janesko, B. G.; Gomperts, R.; Mennucci, B.; Hratchian, H. P.; Ortiz, J. V.; Izmaylov, A. F.; Sonnenberg, J. L.; Williams-Young, D.; Ding, F.; Lipparini, F.; Egidi, F.; Goings, J.; Peng, B.; Petrone, A.; Henderson, T.; Ranasinghe, D.; Zakrzewski, V. G.; Gao, J.; Rega, N.; Zheng, G.; Liang, W.; Hada, M.; Ehara, M.; Toyota, K.; Fukuda, R.; Hasegawa, J.; Ishida, M.; Nakajima, T.; Honda, Y.; Kitao, O.; Nakai, H.; Vreven, T.; Throssell, K.; Montgomery Jr., J. A.; Peralta, J. E.; Ogliaro, F.; Bearpark, M. J.; Heyd, J. J.; Brothers, E. N.; Kudin, K. N.; Staroverov, V. N.; Keith, T. A.; Kobayashi, R.; Normand, J.; Raghavachari, K.; Rendell, A. P.; Burant, J. C.; Iyengar, S. S.; Tomasi, J.; Cossi, M.; Millam, J. M.; Klene, M.; Adamo, C.; Cammi, R.; Ochterski, J. W.; Martin, R. L.; Morokuma, K.; Farkas, O.; Foresman, J. B.; Fox, D. J.; Gaussian 16, Revision A.03, Gaussian, Inc., Wallingford CT, 2016.

27. Marcus, R.-A.; Аnnu. Rev. Phys. Chem. 1964, 15, 155.

28. Marcus, R.-A.; Rev. Mod. Phys. 1993, 65, 599.

29. Barbara, P.-F.; Meyer, T.-J.; Ratner, M.-A.; J. Phys. Chem. 1996, 100, 13148.

30. Cornil, J.; Lemaur, V.; Calbert, J.-P.; Brédas, J.-L.; Adv. Mater. 2002, 14, 726.

31. Lemaur, V.; da Silva Filho, D.-A.; Coropceanu, V.; Lehmann, M.; Geerts, Y.; Piris, J.; Debije, M.-G.; van de Craats, A.-M.; Senthilkumar, K.; Siebbeles, L.-D.-A.; Warman, J.-M.; Bredas, J.-L.; Cornil, J.; J. Am. Chem. Soc. 2004, 126, 3271.

32. Wang, L.-J.; Li, P.; Xu, B.; Zhang, H.-Y.; Tian, W.-J.; Org. Electron 2014, 15, 2476

33. Liang, M.; Yin, J.; Chaitanya, K.; Ju, X.-H.; J. Theor. Comput. Chem. 2016, $15,1650027$.

34. Chen, X.-K.; Zou, L.-Y.; Ren, A.-M.; Fan, J.-X.; Phys. Chem. Chem. Phys. 2011, 13, 19490.

35. Chen, X.-K.; Guo, J.-F.; Zou, L.-Y.; Ren, A.-M.; Fan, J.-X.; J. Phys. Chem. C 2011, 115, 21416.

36. Sun, D.-G.; Ding, F.-J.; Zhao, K. Q.; Acta Chim. Sinica 2008, 66, 738.

37. Farazdel, A.; Dupuis, M.; Clementi, E.; Aviram, A.; J. Am. Chem. Soc. 1990, 112, 4206.

38. Skourtis, S.-S.; Beratan, D.-N.; J. Phys. Chem. B 1997, 101, 1215.

39. Li, Z.-T.; Zhi, L.-J.; Lucas, N.-T.; Wang, Z.-H.; Tetrahedron 2009, 65, 3417.

40. Fyfe, C.-A.; Lewis, A.-R.; J. Phys. Chem. B 2000, 104, 48.

41. Saranya, G.; Navamani, K.; Senthilkumar, K.; Chem. Phys. 2014, 433, 48. 